Ann. Zootech., I97I, 20 (I), 9I-105.

\title{
VALEUR ALIMENTAIRE DE LA LEVURE SULFITIQUE (TORUTIL) ET DU LACTOSÉRUM-LEVURE (S. A. V.) POUR LE RAT : EFFET DE LA SUPPLÉMENTATION PAR LA LYSINE ET LA MÉTHIONINE
}

\author{
F. COLOMER-ROCHER (1) et C. FÉVRIER \\ avec la collaboration technique de M. Lecourtier \\ Station de Recherches sur l'Élevage des Porcs, \\ Centre national de Recherches zootechniques, I. N.R. A. \\ 78 - Jouy-en-Josas
}

\section{RÉSUMÉ}

Au cours d'une expérience chez le Rat on a comparé la valeur de complémentation, vis-à-vis de l'orge, des protéines du tourteau de soja, de la levure sulfitique et du lactosérum levuré, additionnés ou non de DL-Méthionine ou de L-Lysine. Les régimes employés étaient isoénergétiques, isoprotidiques et isocellulosiques. Les animaux recevaient l'aliment et l'eau à volonté.

La vitesse de croissance obtenue avec le régime orge-levure sulfitique a été inférieure de to, 5 p. xoo à celle obtenue avec le régime orge-soja. Ce dernier régime a permis d'obtenir un Coefficient d'Utilisation Protidique supérieur de i r p. roo à celui obtenu avec le régime orgelevure sulfitique, ce qui est confirmé par d'adiposité plus élevée des rats recevant ce régime. L'addition de méthionine au régime orge-levure sulfitique permet au lot correspondant d'obtenir les mêmes performances que le lot orge-soja, tandis que l'addition de lysine se révèle inefficace. Le facteur limitant primaire des protéines de levures sulfitiques est donc la méthionine. Ces résultats sont en accord avec l'examen des pourcentages de déficit en acides aminés de ces deux régimes par rapport aux besoins de Rat.

La vitesse de croissance obtenue avec le régime lactosérum levuré a été inférieure de II,4 $_{4}$ p. roo à celle obtenue avec le régime orge-soja. Ce résultat s'explique par une consommation inférieure de $10,6 \mathrm{p}$. roo dans le même sens, les critères d'efficacité alimentaire et de composition corporelle n'étant pas différents. La réduction de consommation peut être attribuée aux teneurs en lactose et en minéraux du régime lactosérum levuré. Par ailleurs, l'addition de DL-Méthionine ou de L-Lysine à ce régime apporte peu ou pas d'amélioration des caractéristiques de croissance, d'efficacité alimentaire ou de composition corporelle. L'explication de ce phénomène peut être recherchée dans l'insuffisance de l'ingéré énergétique, le rapport énergie/lysine étant alors trop faible.

(1) Adresse actuelle : Minesterio de Agricultura, Centro de Investigacion y desarrollo agrario del Ebro. Montanana I77 (Aula Del) Apartado 202, Saragosse (Espagne). 


\section{INTRODUCTION}

Au cours d'une précédente expérience réalisée sur Porc (COLOMER-Rocher et FÉvrIER, Ig68) il a été constaté que la complémentation des protéines de 1'orge par celle de la levure sulfitique ou par celle du lactosérum levuré ne permettait pas une rétention azotée aussi élevée que celle obtente avec la ccmplémentation par le tourteau de soja.

Dans le cas de la levure sulfitique, les résultats expérimentaux semblent en accord avec l'analyse en acides aminés de cet aliment. Par contre, dans le cas du lactosérum levuré, il n'en est pas de même et nous avons été amenés à émettre l'hypothèse suivant laquelle le traitement thermique provoquerait une indisponibilité de certains acides aminés du lactosérum levuré, et notamment de la lysine.

La valeur nutritionnelle d'une protéine est, en effet, fondée non seulement sur la proportion d'acides aminés qu'elle renferme, mais aussi sur leur disponibilité, or celle-ci dépend pour une grande part des traitements thermiques subis par 1'aliment. Ainsi, la chaleur sèche peut créer de nouvelles liaisons résistantes àl'action des enzymes, entre les fonctions aldéhyde ou cétone des glucides et les groupements $\mathrm{NH}_{2}$ de la lysine contenue dans une protéine (MAIL,ARD, I905; BLOCK et al., I946). Dans les aliments soumis à une surchauffe ou à une cuisson trop prolongée, les acides diaminés sont scuvent plus dénaturés que les autres, mais ceci ne constitue pas une règle absolue (PETIT et ADRIAN, I967), le chauffage rend donc indisponible pour l'organisme une partie de certains acides aminés présents dans l'aliment.

Dans le cas des levures-aliments qui subissent des traitements thermiques en cours de fabrication, l'indisponibilité des acides aminés peut être importante puisque les résidus sur lesquels les levures sont cultivées sont riches en sucres réducteurs. JACQUOT (I946) a montré que la disponibilité des acides aminés dans les levures varie avec les modalités de leur préparation. Toutefois, des expériences récentes réalisées avec de la levure sulfitique montrent que la lysine de cet aliment est remarquablement résistante aux traitements thermiques (ADRIAN et FRANGNE, I970).

Pour vérifier notre hypothèse initiale nous avons étudié sur le Rat 1'effet d'une addition de lysine et de méthionine aux régimes orge-levure sulfitique et orgelactosérum levuré, précédemment utilisés sur le Porc.

\section{MATÉRIEL ET MÉTHODES}

Le régime orge-soja est utilisé comme témoin par rapport aux régimes orge-levure sulfitique ou orge-lactosérum levuré, additionnés ou non de Lysine et de Méthionine. L'expérience a porté sur 9 lots dont les nomenclatures et les niveaux de supplémentation en acides aminés sont rapportés à la page suivante.

Le taux de protéines pour tous les régimes était de $\mathrm{r}_{3} \mathrm{p}$. Ioo dont $52 \mathrm{p}$. Ioo étaient fournis par celles de l'orge, et $4^{8} \mathrm{p}$. Ioo par la protéine à tester. Les teneurs en énergie brute, en cellulose, en $\mathrm{Ca}$ et $\mathrm{P}$ étaient équivalentes dans tous les régimes dont la composition et les résultats d'analyse sont donnés aux tableaux I et 2 .

Cette expérience sur Rat étant préliminaire à une expérience sur Porc, le taux de o, I p. Ioo de méthionine supplémentaire a été choisi car il permet d'atteindre un taux de 0,53 p. Ioo, ce 


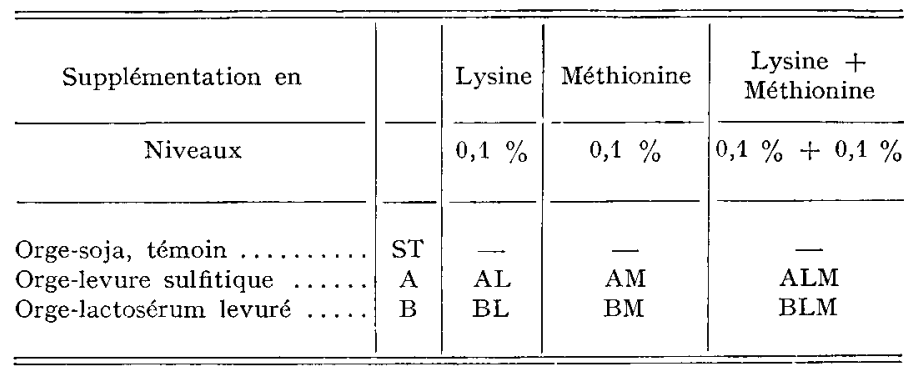

TABLEAU I. - Composition des régimes, en pourcentage

TABLE I. - Composition of diets, per cent

\begin{tabular}{|c|c|c|c|c|}
\hline Régime & $\begin{array}{l}\text { Orge-soja } \\
\quad \text { ST } \\
\text { Barley-soya }\end{array}$ & \begin{tabular}{|c|} 
Orge-levure \\
sulfitique \\
$\mathrm{A}$ \\
Bavley-sulfite yeast
\end{tabular} & $\begin{array}{c}\text { Orge-lactosérum } \\
\text { levuré } \\
\mathrm{B} \\
\text { Barley-yeasted } v \text { they }\end{array}$ & Diet \\
\hline 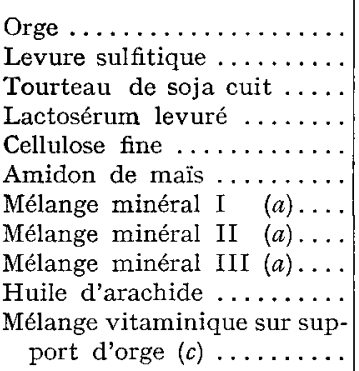 & $\begin{array}{l}59,00 \\
- \\
16,20 \\
- \\
4,00 \\
11,98 \\
\frac{3,82}{-} \\
1,00 \\
4,00\end{array}$ & $\begin{array}{c}59,00 \\
13,00 \\
- \\
\overline{5,00} \\
14,18 \\
3,82 \\
- \\
1,00 \\
4,00\end{array}$ & $\begin{array}{l}59,00 \\
- \\
\overline{25,00} \\
5,00 \\
5,26 \\
- \\
\overline{0,74} \\
1,00 \\
4,00\end{array}$ & $\begin{array}{l}\text { Barley } \\
\text { Sulfite yeast } \\
\text { Soyabean meal } \\
\text { Yeasted whey } \\
\text { Cellulose } \\
\text { Corn starch } \\
\text { Salt mixture I } \\
\text { Salt mixture II } \\
\text { Salt mixture III } \\
\text { Peanut oil } \\
\text { Vitamines, mixed in } \\
\quad \text { barley }\end{array}$ \\
\hline Total & 100,00 & 100,00 & 100,00 & Total \\
\hline
\end{tabular}

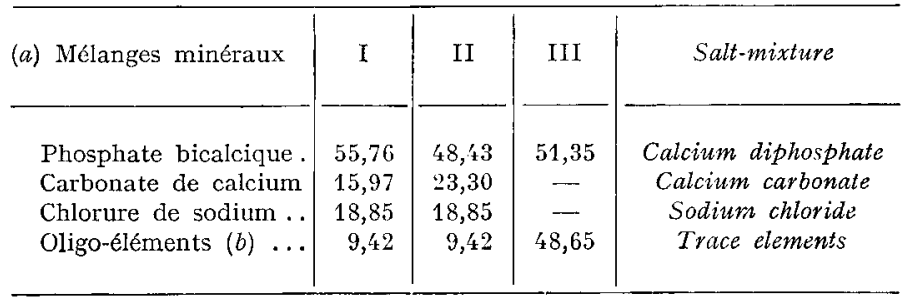

(b) Carbonate magnésium 83,33 ; sulfate ferreux 8,33 ; sulfate manganèse 2,50 ; sulfate de cuivre 0,84 ; sulfate zinc 4,16 ; sulfate cobalt 0,42 ; iodure de potassium 0,42 .

(c) Composition p. 100 : vit. A $500000 \mathrm{UI}$; vit. $\mathrm{D}_{2} 50000 \mathrm{UI}$, riboflavine $200 \mathrm{mg}$; acide pantothénique $500 \mathrm{mg}$; choline $100 \mathrm{mg}$; vit. $\mathrm{B}_{12} 1 \mathrm{mg}$. 
F. COLOMER-ROCHER, C. FÉVRIER

TABLEAU 2. - Composition chimique des régimes

TABLE 2. - Gross chemical composition of Diets

\begin{tabular}{|c|c|c|c|c|}
\hline Régime & $\begin{array}{c}\text { Orge-soja } \\
\text { ST }\end{array}$ & $\begin{array}{c}\text { Orge-levure } \\
\text { sulfitique } \\
\text { A }\end{array}$ & $\begin{array}{c}\text { Orge-lactosérum } \\
\text { levuré } \\
\text { B }\end{array}$ & Diet \\
\hline $\begin{array}{l}\text { P. } 100 \text { du régime } \\
\text { Matière sèche } \ldots \ldots \ldots \ldots \\
\text { Matières minérales } \ldots \ldots \ldots \ldots \\
\text { Matières grasses } \ldots \ldots \ldots \ldots \\
\text { Mat. azotées }(N \times 6,25) \ldots \\
\text { Cellulose brute }(\text { calculée) } \ldots \\
\text { Extractif non azoté } \ldots \ldots \ldots \\
\text { Énergie brute, cal/g de } \mathrm{MS} \\
\text { Calcium } \ldots \ldots \ldots \ldots \ldots \ldots \ldots \\
\text { Phosphore } \ldots \ldots \ldots \ldots \ldots \ldots\end{array}$ & $\begin{array}{c}87,40 \\
5,22 \\
2,61 \\
13,50 \\
10,00 \\
56,07 \\
4,289 \\
0,800 \\
0,750\end{array}$ & $\begin{array}{c}87,56 \\
5,19 \\
2,20 \\
12,71 \\
10,17 \\
57,29 \\
4,332 \\
0,800 \\
0,750\end{array}$ & $\begin{array}{c}87,51 \\
6,51 \\
2,60 \\
12,96 \\
10,01 \\
55,40 \\
4,303 \\
0,800 \\
0,750\end{array}$ & $\begin{array}{l}\text { P. } 100 \text { of diet } \\
\text { Dry matter } \\
\text { Minerals } \\
\text { Lipids } \\
\text { Crude protein } \\
\text { Crude fiber } \\
\text { Protein free extract } \\
\text { Energy cal/g DM } \\
\text { Calcium } \\
\text { Phosphorus }\end{array}$ \\
\hline
\end{tabular}

qui semble être suffisant pour le Porc en croissance, le besoin du Rat étant plus élevé (tabl. 3). Dans le cas de la lysine, on a supposé une indisponibilité de 20 p. Ioo pour celle du lactosérum levuré, ce qui a conduit à choisir un taux de o, I p. Ioo également. La composition des régimes en acides aminés a été calculée à partir de la composition des constituants, déterminée par PIoN et Fauconneau (I966) pour l'orge et le tourteau de soja, et par Pion (1967) pour la levure sulfitique et le lactosérum levuré (tabl. 3).

TABLEAU 3. - Besoins en acides aminés des rats en croissance (NRC, 1962) et composition en acides aminés des régimes, exprimés en p. 100

TABLE 3. - Aminoacids requirements of the rat $(N R C, 1962)$ and aminoacid composition of diets ( $p$. cent)

\begin{tabular}{|c|c|c|c|c|}
\hline Acides aminés & $\begin{array}{l}\text { Besoins } \\
\text { NRC } \\
(1962)\end{array}$ & $\begin{array}{l}\text { Régime } \\
\text { Orge-levure } \\
\text { sulfitique } \\
\text { ST }\end{array}$ & $\begin{array}{c}\text { Régime } \\
\text { Orge-soja } \\
\text { A }\end{array}$ & $\begin{array}{c}\text { Régime } \\
\text { Orge-lactosérum } \\
\text { levuré } \\
\text { B }\end{array}$ \\
\hline Histidine $\ldots \ldots \ldots \ldots \ldots \ldots$ & 0,30 & 0,27 & 0,32 & 0,27 \\
\hline Lysine $\ldots \ldots \ldots \ldots \ldots \ldots$ & 0,90 & 0,73 & 0,69 & 0,78 \\
\hline Leucine ................. & 0,80 & 0,88 & 1,00 & 1,06 \\
\hline Isoleucine $\ldots \ldots \ldots \ldots \ldots \ldots$ & 0,50 & 0,55 & 0,60 & 0,62 \\
\hline Phénylalanine ............. & $0,90(1)$ & 0,59 & 0,72 & 0,58 \\
\hline Tyrosine $\ldots \ldots \ldots \ldots \ldots$ & - & 0,44 & 0,49 & 0,47 \\
\hline Méthionine $\ldots \ldots \ldots \ldots \ldots \ldots$ & $0,60\left(^{2}\right)$ & 0,21 & 0,21 & 0,24 \\
\hline Cystine $\ldots \ldots \ldots \ldots \ldots \ldots$ & - & 0,22 & 0,27 & 0,27 \\
\hline Méthionine + Cystine ........ & - & 0,43 & 0,48 & 0,51 \\
\hline Threonine $\ldots \ldots \ldots \ldots \ldots \ldots$ & 0,50 & 0,51 & 0,50 & 0,62 \\
\hline Valine $\ldots \ldots \ldots \ldots \ldots \ldots$ & 0,70 & 0,70 & 0,73 & 0,73 \\
\hline Arginine $\ldots \ldots \ldots \ldots \ldots \ldots$ & 0,20 & 0,63 & 0,84 & 0,54 \\
\hline
\end{tabular}

(1) Un tiers peut être remplacé par la tyrosine.

(2) Un tiers à un demi peut être remplacé par la cystine. 
Les rats mâles, au sevrage, étaient de souche Wistar $C F$ et d'un poids de $50 \mathrm{~g}$. Quatre-vingtdix rats ont été répartis en 9 lots de ro animaux, suivant la méthode des blocs, en fonction de leur poids et de leur gain de poids pendant une période préexpérimentale d'une semaine.

Chaque animal a reçu l'aliment ad libitum sous forme de farine sèche durant le temps nécessaire pour obtenir un gain de poids de $190 \mathrm{~g}$.

A la fin de la période expérimentale, après un jeûne de 6 à 8 heures, les animaux ont été asphyxiés au gaz d'éclairage puis conservés au congélateur à -- $20^{\circ} \mathrm{C}$. Les carcasses entières ont été ensuite broyées et homogénéisées à l'aide d'un hachoir rotatif "Robot coupe ». Les teneurs en matière sèche, en azote et en cendres ont été déterminées et l'on a admis que les lipides étaient représentés par la fraction résiduelle du poids total.

Les valeurs moyennes obtenues pour chaque lot et les critères étudiés ont été analysés suivant le test des moyennes ordonnées (Harter, 1960).

\section{RÉSULTATS}

\section{r. - Comparaison des régimes non supplémentés}

Le régime orge-soja a permis une vitesse de croissance supérieure à celle obtenue avec les deux autres régimes $(5,3 \mathrm{I} \mathrm{g}$ contre $4,84 \mathrm{~g}$ et $4,48 \mathrm{~g})$. Dans le cas de la levure sulfitique, la vitesse de croissance des rats est significativement moins rapide lorsque l'on compare les résultats après 28 jours d'expérience (tab1. 4 ) soit $5,58 \mathrm{~g}$ pour le régime soja, 5,I4 pour la levure sulfitique, mais les vitesses ne sont plus significativement différentes lorsque l'on considère le même gain de poids de Igo g (tabl. 5). Par rapport au soja, la durée d'expérience n'est augmentée que de 4 jours pour la levure sulfitique au lieu de 7 jours avec le lactosérum levuré.

Les rats recevant le lactosérum levuré ont fait prenve d'un appétit nettement inférieur à celui observé avec les deux autres aliments ( $\mathrm{I} 5,8 \mathrm{~g} / \mathrm{j}$ contre $\mathrm{I} 7,7$ et $\mathrm{I} 7,9$ ), mais les quantités totales d'aliment consommées pour obtenir un même gain de poids ont été identiques dans les deux régimes soja et lactosérum levuré ( $646 \mathrm{~g}$. et $683 \mathrm{~g}$.) et légèrement supérieures avec la levure sulfitique (709 g.), mais cette différence n'est pas significative (tabl. 5).

La composition corporelle des rats recevant les régimes soja ou lactosérum levuré a été identique : par contre, les rats qui ont reçu le régime levure sulfitique ont présenté une adiposité significativement plus élevée que les rat recevant les régimes précédents, et leur teneur en protéines a été significativement inférieure à celle des rats recevant le lactosérum levuré (tabl.6). En revanche, la teneur en minéraux a été identique dans les trois lots d'animaux.

Le Coefficient d'Utilisation Pratique de l'azote, c'est-à-dire la proportion d'azote retenu vis-à-vis de l'azote ingéré orge-soja $(38,7 \mathrm{p}$, Ioo) pour le régime a été supérieur à ceux des autres régimes $(34,6 \mathrm{p}$. roo et $37,5 \mathrm{p}$. roo) mais la différence n'est significative que dans le cas de la levure sulfitique. Par ailleurs, on n'observe pas de différences concernant le coefficient d'efficacité protidique et la rétention calorique, c'est-à-dire du pourcentage de l'énergie retenue par rapport à l'énergie consommée (tabl. 7).

\section{2. - Effet de la supplémentation en acides aminés du régime orge-levure sulfitique}

L'addition de lysine au régime orge-levure sulfitique s'est révélée être sans effet sur les performances et la composition corporelle des rats qui ont consommé ce régime. On note cependant une diminution de l'ingéré sec et une légère amélioration du 
F. COLOMER-ROCHER, C. FÉVRIER

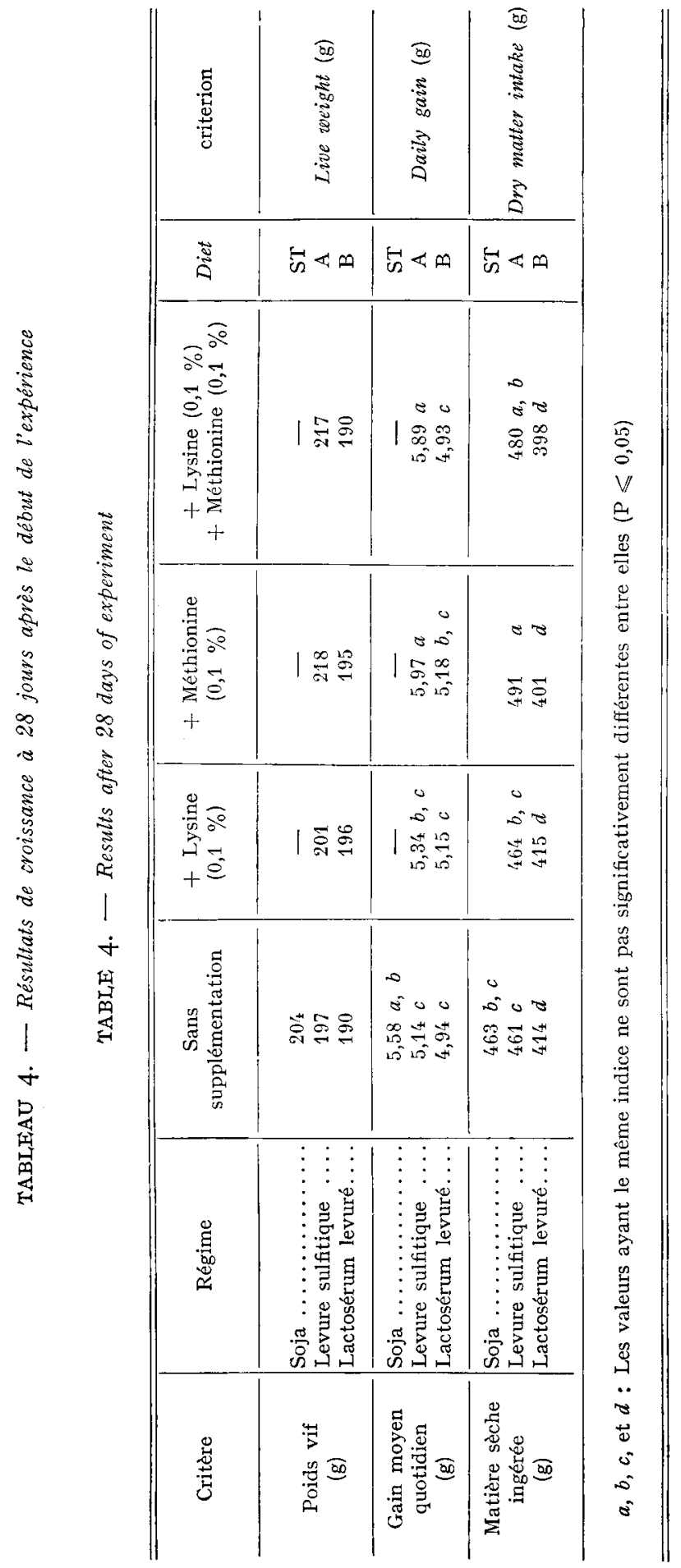


TORUTIL ET S. A. V. : VALEUR PROTÉIQUE CHEZ IE RAT

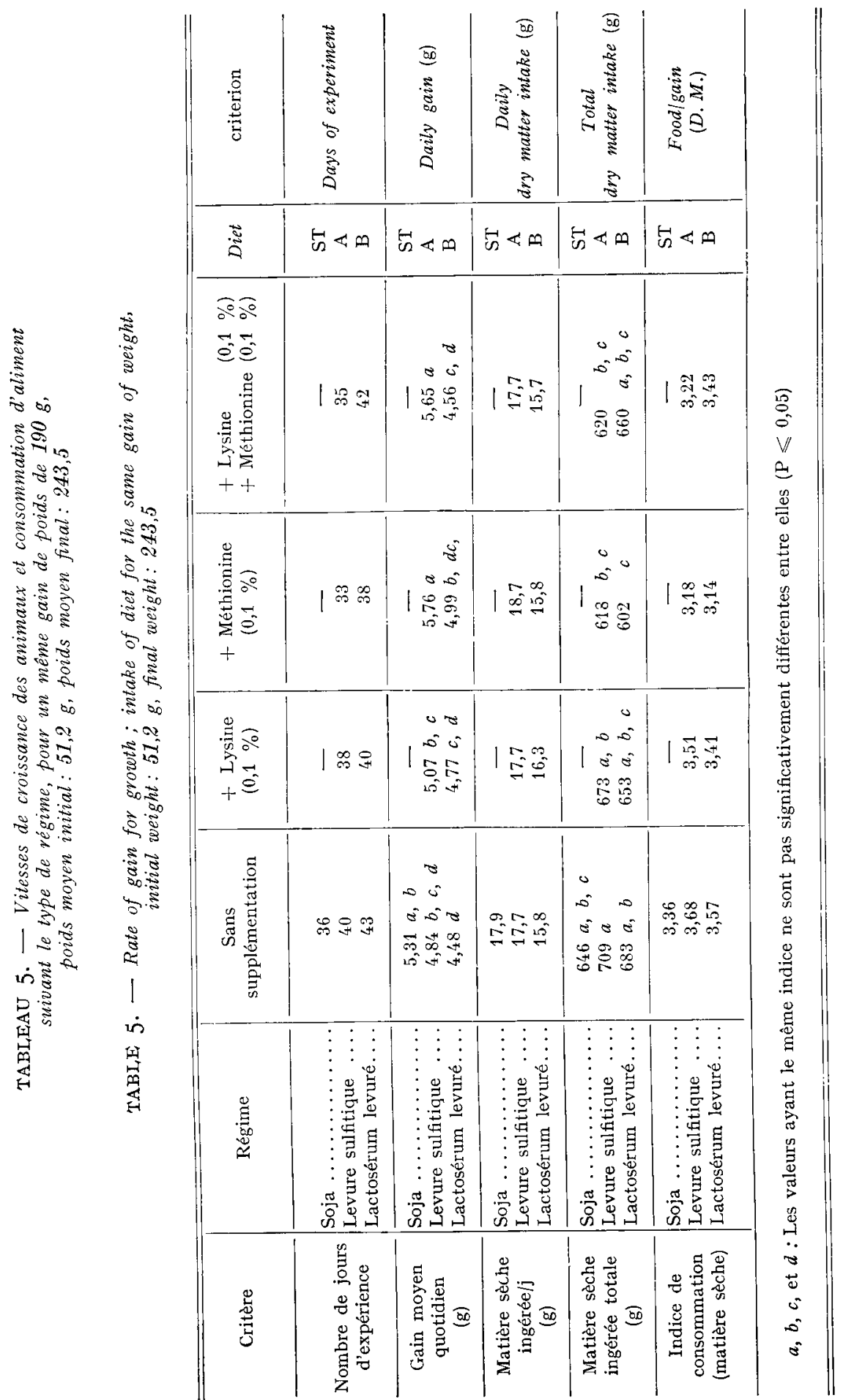


F. COLOMER-ROCHER, C. FÉVRIER

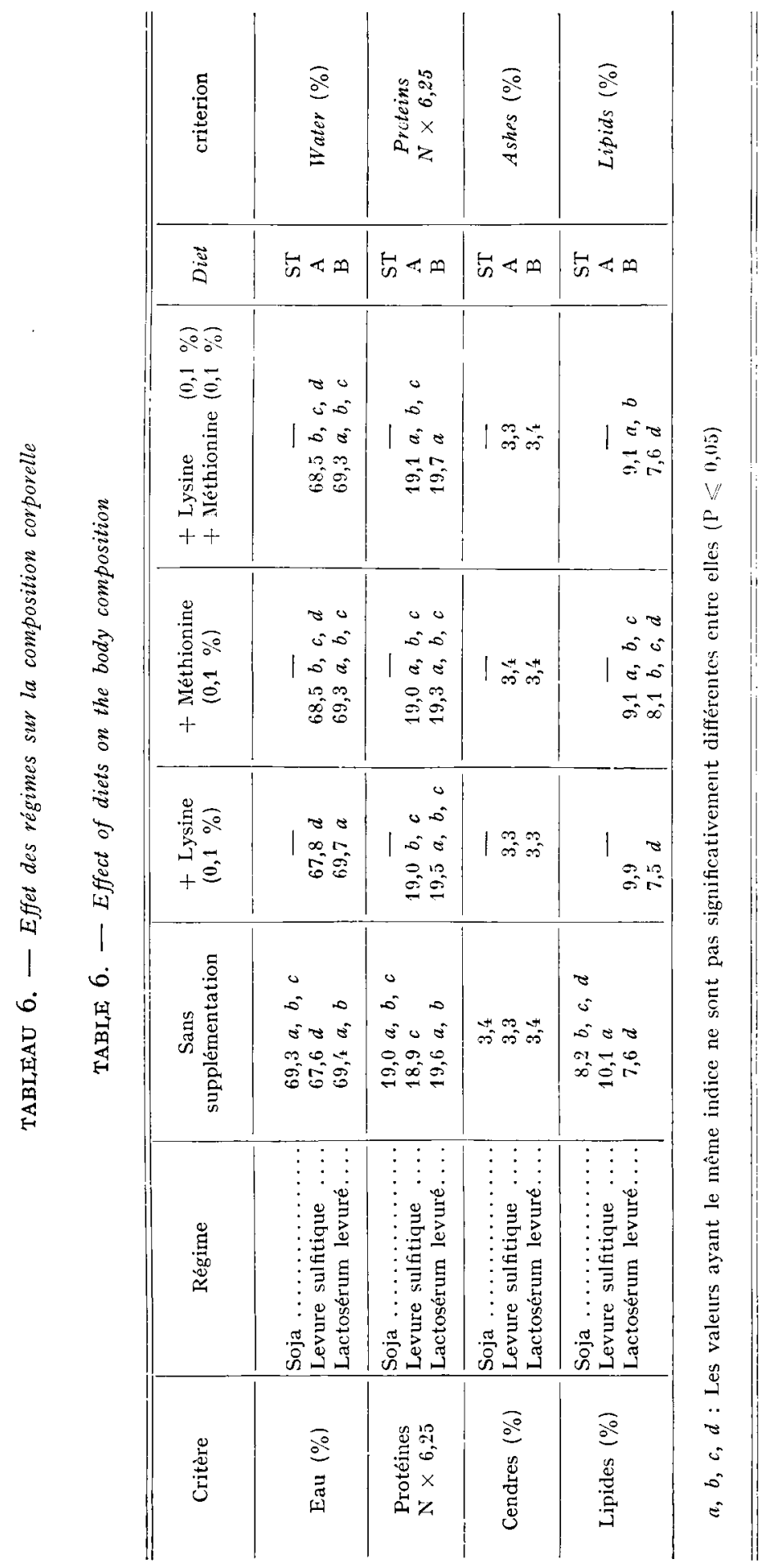


TORUTIL ET S. A. V. : VALEUR PROTÉIQUE CHEZ LE RAT

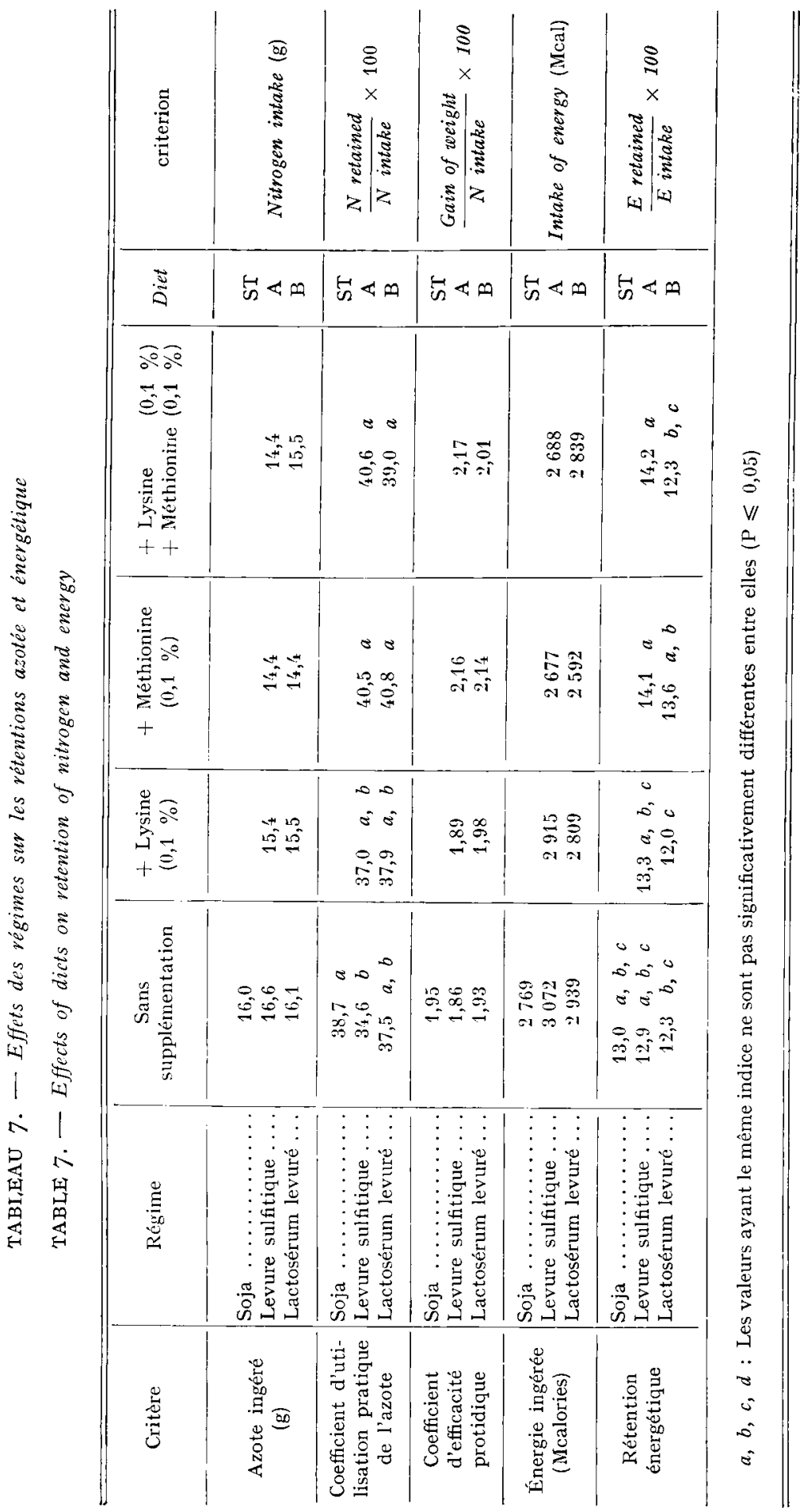


Coefficient d'Utilisation Pratique de l'azote, mais ces variations ne sont pas significatives (tabl. 7).

L'addition de méthionine, en permettant une croissance plus rapide et une consommation totale d'aliment plus faible, a permis une amélioration importante de la rétention azotée qui alors est supérieure à celle provoquée par le régime soja. Ainsi le Coefficient d'Utilisation Pratique de l'azcte augmente de 34,6 à 40,5 p. Ioo, soit en valeur relative de I7, I p. roo. Ceci s'est accompagné d'une faible augmentation non significative, de l'utilisation énergétique du régime. Cependant, au niveau de la composition corporelle les différences, bien que faibles, ont permis de rejoindre celle des animaux témoins : l'adiposité a légèrement diminué tandis que la teneur en protéines a augmenté très légèrement (tabl. 6).

La double supplémentation en lysine et méthionine n'a permis aucune modification des performances ou de la composition corporelle par rapport à la simple supplémentation en méthionine.

Il faut noter enfin que les résultats concernant la vitesse de croissance et l'efficacité alimentaire ont évolué de la même manière en fonction du régime lorsque les comparaisons ont été faites soit pour une même période de 28 jours (table 4 et 5 ), soit pour un même gain de poids de Igo g.

\section{3. - Effet de la supplémentation en lysine et méthionine du régime orge-lactosérum levuré}

L'addition de lysine au régime orge-lactosérum levuré n'a provoqué aucune modification des performances ou de la composition corporelle des rats nourris avec ce régime par rapport à ceux nourris avec le régime initial, et la rétention azotée n'a pas été modifiée. En revanche, la méthionine ajoutée seule a permis une légère augmentation (Ir p. xoo) de la vitesse de croissance qui a alors atteint un niveau peu différent de celui obtenu avec le soja ; corrélativement, l'indice de consommation a diminué significativement de I3,4 p. roo. La composition corporelle des rats n'a pas été modifiée par l'addition de méthionine mais le CUP de l'azote a augmenté de 8,8 p. Ioo. La diffétence n'est toutefois pas significative et le CUP a été identique à celui obtenu avec la levure sulfitique supplémentée par la méthionine $(40,5 \mathrm{p}$. Ioo et 40,8 p. IOo), mais supérieure à celle obtenue avec le soja $(38,7$ p. IOo). Cependant, l'influence favorable d'une addition de méthionine est incertaine puisqu'elle ne s'observe plus en présence d'une addition simultanée de lysine.

Le comportement des deux régimes levurés vis-à-vis de l'addition d'acides aminés est donc assez différent. L'addition de lysine s'est révélée inefficace pour les deux régimes. Par contre, la supplémentation en méthionine a été extrêmement favorable pour le régime levure sulfitique, les performances obtenues étant alors supérieures à celles permises par le soja. Dans le cas du lactosérum levuré, pour lequel on observe une appétibilité plus faible que pour les deux autres régimes, la supplémentation par la méthionine n'a apporté qu'une amélioration incertaine. 


\section{DISCUSSION}

\section{Comparaison des régimes non supplémentés}

Les rats recevant le régime levure sulfitique ont eu une croissance inférieure de Io p. Ioo à celle des rats recevant le soja, bien que la consommation quotidienne ait été identique. Le fait que la consommation totale, pour obtenir un gain de poids de Igo g, soit légèrement supérieure, peut être attribué, en partie, au fait que la durée d'entretien a été plus longue de 4 jours avec la levure sulfitique. La valeur de complémentation des protéines de soja vis-à-vis de l'orge a donc été supérieure à celle de la levure et ceci est en accord avec le fait que le déficit primaire en acides aminés soufrés est plus important dans la levure sulfitique que dans le soja (tab1. 8).

\footnotetext{
TABLEAU 8. - Pourcentage de défucit en acides aminés des régimes par rapport aux besoins du rat (NRC, I962)

TABIE 8. - Percentage of defucit in aminoacids of diets with respert to the requirements for the rat (NRC, I962)
}

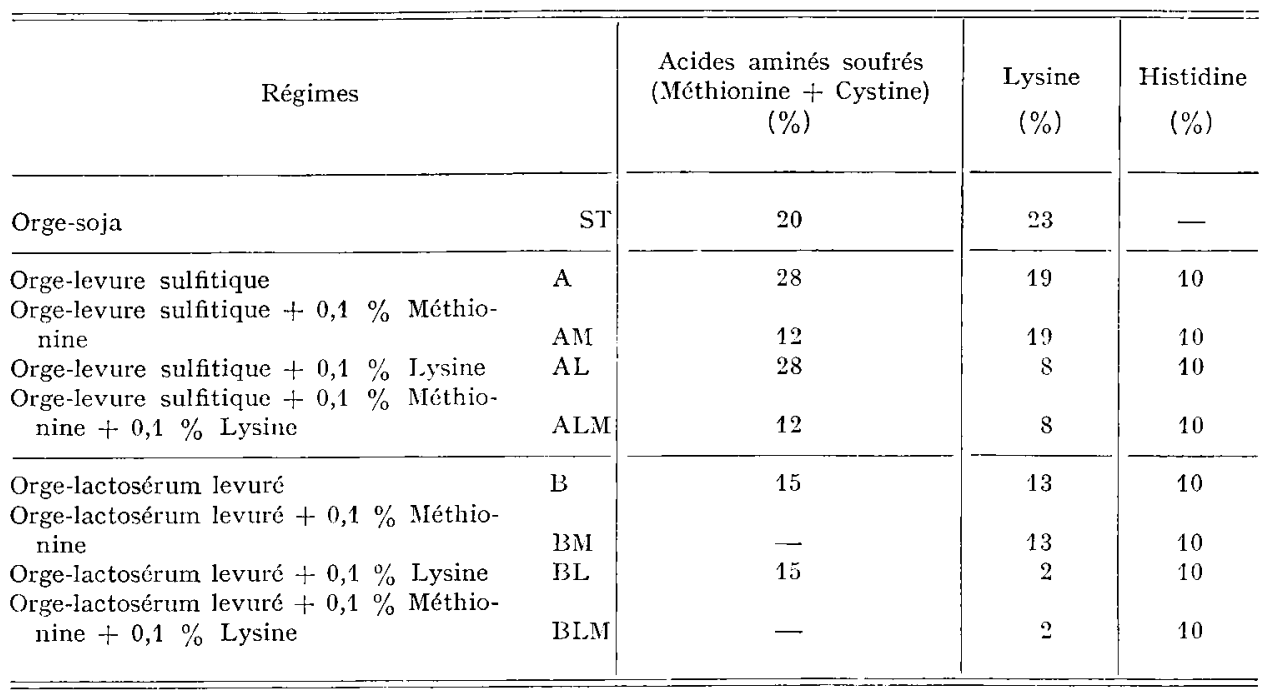

Avec le régime lactosérum levuré, la consommation quotidienne est abaissée de $10,6 \mathrm{p}$. Ioo et la croissance est ralentie de II,4 p. Ioo par rapport au régime soja ; le premier fait peut expliquer le second. En effet, les quantités totales consommées pour obtenir un gain de poids de Igo g ne sont pas significativement différentes. Le léger écart obtenu en défaveur du lot " lactosérum levuré " pourrait seulement traduire la dépense d'entretien supplémentaire de 7 jours. Par ailleurs, les coefficients d'utilisation pratique de l'azote, les coefficients de rétention énergétique et les compositions corporelles ont des valeurs comparables. Or, d'après l'analyse des déficits 
en acides aminés (tabl. 8), le lactosérum levuré devrait avoir une valeur de complémentation vis-à-vis de l'orge supérieure à celle du soja. Le facteur limitant est donc la faible consommation quotidienne.

Deux faits peuvent expliquer la moindre appétence du régime lactosérum levuré. On calcule que la ration contient $5,5 \mathrm{p}$. Ioo de lactose, or la consommation d'un régime diminue linéairement lorsque le taux de lactose augmente (FÉVRIER et RÉRAT, I964). Par ailleurs, la quantité des minéraux contenue dans ce même régime est supérieure à celle contenue dans le régime "orge-soja " par suite de la teneur élevée de ces constituants et notamment du potassium, dans le lactosérum levuré. Or, selon DANIEL et HARVEY (I947), l'apport excessif de minéraux du lactosérum réduit la croissance chez le Rat.

La comparaison des régimes levure sulfitique et lactosérum levuré résulte des conclusions des deux comparaisons précédentes avec le soja. En effet, la similitude des vitesses de croissance observée avec ces deux régimes s'explique par le fait que la meilleure appétence de la levure sulfitique a compensé sa moins bonne efficacité alimentaire par rapport au lactosérum levuré.

\section{Effet de la supplémentation du régime orge-levure sulfitique par les acides aminés}

L'addition de 1ysine au régime orge-levure sulfitique n'entraîne aucune modification de la consommation ou de la vitesse de croissance. L'efficacité alimentaire n'est donc pas modifiée. De plus la composition corporelle des animaux ne varie pas. Ia lysine ne constitue donc pas le facteur limitant primaire de ce régime, ce qui est en accord avec l'analyse en acides aminés (tabl. 8).

Avec l'addition de méthionine, l'augmentation de $6,5 \mathrm{p}$. Ioo de la quantité d'aliment consommée au bout de 28 jours ne peut expliquer à elle seule l'amélioration de croissance de $\mathrm{r} 6, \mathrm{I}$ p. Ioo observée. La valeur biologique des protéines du régime est donc améliorée. On constate en effet que la quantité d'aliment nécessaire pour un gain de poids de Igo g diminue significativement de $\mathrm{I}_{4,7} \mathrm{p}$. Ioo pour le lot supplémenté. Ceci est confirmé par l'amélioration très nette, et significative, du coefficient d'utilisation pratique de l'azote. Les observations concernant la composition corporelle des animaux vont dans le même sens, bien que la baisse d'adiposité soit faible.

Ces résultats sont en contradiction avec ceux de CAIET et al. (I962) obtenus sur rats, et dans lesquels l'addition de méthionine seule n'améliorait ni la croissance, ni l'efficacité protidique du régime à base de levure, la teneur en lysine étant alors insuffisante. En revanche, les mêmes auteurs constataient l'efficacité de la supplémentation par la méthionine d'un régime levuré pour Poulet. Par ailleurs, le régime " orge-levure ", utilisé dans l'expérience présente a pu être supplémenté avec succès chez le Porc (F́́vriér C. et SÈvE, données non publiées). Ces différences avec les résultats de CALET et al., peuvent s'expliquer par la différence de nature de la levure et par l'évolution de la technologie qui évite maintenant des phénomènes pouvant entraîner une indisponibilité de la lysine, et par la diffétence des taux azotés utilisées : I4 p. IOO au lieu de I3 p. Ioo dans la présente expérience.

Ia double supplémentation du régime en lysine et méthionine a n'a pas été plus efficace que la seule supplémentation en méthionine, et les chiffres obtenus 
sont pratiquement identiques. Il est donc possible que le taux des acides aminés soufrés n'ait pas été suffisamment relevé pour que la supplémentation en lysine s'exprime. Mais, il est également possible que la lysine ne soit pas le facteur limitant secondaire du régime et que sa disponitilité soit satisfaisante (ADRIAN et FrangNE, I970).

\section{Effet de la supplémentation en acides aminés du régime orge-lactosérum-levuré}

La seule amélioration que l'on peut noter concerne l'effet de la supplémentation en méthionine sur l'efficacité alimentaire, mais cette amélioration disparaît dans le lot où l'on supplémente simultanément en méthionine et lysine.

L'inconstance de la réponse à la supplémentation en 1ysine et méthionine peut s'expliquer de la façcn suivante : la consommation constatée dans tous les régimes contenant du lactosérum levuré supplémenté ou non, a été inférieure de plus de Io p. Ioo à celle observée dans les régimes contenant du soja ou de la levure sulfitique. Comme nous l'avons déjà indiqué, les teneurs en lactose et en minéraux sont probablement en cause. La réduction de consommation entraîne une insuffisance de l'ingéré énergétique. Or, d'après les résultats de LERNER (I968), il y a interaction énergie $\times$ lysine chez le Rat. Pour un taux de lysine donné, il existe un niveau d'énergie optimum en dessous duquel le coefficient d'utilisation pratique de l'azote diminue. Ce résultat avait déjà été constaté chez le Porc par AbERnathy et al.(I958) et MrTCHEI, et al. (I965).

Dans l'expérience présente, le taux d'énergie est resté constant et sans doute en dessous de l'optimum ; ce qui a fait que 1'addition de lysine avec ou sans méthionine supplémentaire n'a pu être valorisée, et que l'on n'a constaté aucune amélioration par rapport au témoin. L'hypothèse que nous avions formulée à la suite de notre expérience chez le Porc resterait donc à étudier avec un niveau énergétique du régime plus élevé.

\section{CONCLUSION}

Un régime orge-levure sulfitique présente une efficacité alimentaire inférieure à celle d'un régime orge-soja. Cette différence est due à un équilibre défavorable des acides aminés vis-à-vis des besoins dı Rat. Une addition de DL-méthionine à raison de $\mathrm{o}, \mathrm{I}$ p. Ioo du régime permet d'obtenir une efficacité alimentaire égale à celle du régime orge-soja et une addition supplémentaire de L-1ysine n'apporte aucune amélioration. On en conclut donc que le facteur limitant primaire du régime orge-levure sulfitique est bien constitué par les acides aminés soufrés, conformément à l'analyse et que la lysine de la levure est peu détériorée.

En revanche, si le régime orge-lactosérum levuré présente une efficacité alimentaire inférieure à celle du régime orge-soja, le facteur limitant primaire ne semble pas être dû̀ à un déséquilibre en acides aminés mais à un facteur d'inappétence, ce qui limite la consommation d'énergie par l'animal et ne permet pas de mettre en évidence un effet bénéfique d'une supplémentation en acides aminés. La nature du facteur limitant de 1'utilisation du lactosérum levuré (S. A. V.) chez le Rat reste encore indéterminée. 


\title{
SUMMARY
}

\author{
FEEDING VALUE OF SULPHITE YEAST AND YEAST-TREATED \\ WHEY (S. A. V .) FOR THE RAT : EFFECT OF LYSINE \\ AND METHIONINE SUPPLEMENTATION
}

During an experiment on weaned White Wistar rats, we studied the protein of sulphite yeast (A) and yeast-treated whey (S. A. V.) (B) as a to supplement barley, with or without addition of L-lysine (L) and DL-methionine (L), and in comparison with a control diet containing barleysoybean oil meal (ST) without addition of amino acids. The utilization of these diets by the pig (COLOMER-ROCHER and FEVRIER, I968) led to the assumption that part of the lysine from yeasttreated whey could be non available and that the first limiting factor of the two diets was methionine. The diets were isocaloric, isoproteic and isocellulosic (tables 1,2 and 3 ); the rats, fed and housed individually, received water and feed ad libitum.

The growth rate obtained with the diet barley-sulphite yeast (A) was inferior (I0,5 p. Ioo) to that obtained with the diet barley-soybean (ST) (table 4 ). The protein efficiency coefficient ( $N$ retained/ $N$ ingested) obtained with this last diet (ST) was I I p. Ioo higher than that resulting from the barley-sulphite yeast diet (A) (table 7), which was confirmed by a higher degree of adiposity in the rats receiving diet A compared with those receiving diet ST (table 6 ).

The addition of methionine to the barley-sulphite yeast diet (AM) gave the same performances as those obtained with the barley-soybean diet (ST), whereas the addition of lysine (AL) appears to be ineffective. Thus, sulphur amino acids are the first limiting factor of sulphite yeast. The results are in agreement with the deficit in amino acids of the diets A, AL, ALM and ST in comparison with the requirements of the rat (N. R. C. I962).

When using yeast-treated whey $(B)$, the growth rate was inferior ( $I, 4$ p. Ioo) to that obtained with soybean (ST) (table 4 ). This result can be explained by the fact that the consumption of $B$ was Io,6 p. Ioo lower than that of ST; the criteria of food efficiency and body composition did not differ. The decrease in consumption may be due to the lactose and mineral contents of the yeasttreated whey diet. Moreover, the addition of DL-methionine or L-lysine to the diet producesa small (BM) or no (BLM-BL) improvement of growth rate, food efficiency or body composition. The lack of response, especially when adding lysine, may however be explained by an insufficient energy supply, since a too low food intake results in a too low energy/lysine ratio.

\section{RÉFÉRENCES BIBLIOGRAPHIQUES}

Adrian J., Frangne R., I970. Valeur protidique de la levure Torula en fonction des modalités thermiques du chauffage. Ind. Alim. agr., 87, 393-399.

Abernathy R. P., Sewell R. F., TARPley R. L., I958. Interrelationships of protein, lysine and energy in diets of growing swine. J. Anim. Sci., 17, 635-639.

Block R. J., Jones D. B., Gersdorff C. E. F., 1934. The effect of dry heat and dilute alkali on the lysine of casein. J. Biol. Chem., 105, 667-668.

Calet C., Adrian J., Jacouot R., I962. Étude de la valeur nutritionnelle de la levure utilisée sous trois formes différentes : levure sèche, levure autolysée et autolysat de levure. Ann. Zootech., 11, IO3-II4.

Colomer-Rocher F., FÉvrier C., I968. Valeur alimentaire des protéines de la levure sulfitique et du lactosérum chez le Porc en croissance. Ann. Zootech., 17, 409-427.

Daniel F. K., Harvey E. H., I947. Some observations on the nutritional value of dialyzed whey solids. $J$. Nutr., 33, 429-436.

EldRed N. R., Rodney G., r946. The effect of proteolytic enzymes on raw and heated casein. $J$. Biol. Chem., 162, 26r-265.

Février C., Rérat A., I964. Influence du lactose sur la croissance et sur la composition corporelle du Rat blanc. I. Relations avec le taux protidique de la ration. Ann. Biol. anim. Bioch. Biophys., 4, 423.439.

Fívrier C., Sk̀ve B., Ig69. (Données non publiées.)

Harter H. L., 1960. Critical values for Duncan's nex multiple range test. Biometrics, 16, 677-680. 
JАсQUот R., 1946. Valeur alimentaire de quelques protides de remplacement. Bull. Soc. Sci. Hyg. Aliment., 33, 799-805.

LeRner J. T., Ig68. Contribution à l'étude du besoin de lysine en relation avec le taux énergétique chez le Rat et le Porc. Thèse de Doctorat $3^{\mathrm{e}}$ cycle, Faculté des Sciences, Paris.

Maillard L.-C., Igiz. Action des acides aminés sur les sucres : formation des mélanoìdines par voie méthodique. C. R. Acad. Sci., 154, 66.

Mitchell J. R., Becker D. E., Jensen A. H., Norton H. W., Harmon B. G., 1965. Calorific density of the diet and lysine need of growing swine. J. Anim. Sci., 24, 977-980.

N. R. C., I962. Nutrient requirements' of laboratory animals. National Academy of Sciences. Publication 990.

Petit L., Adrian J., 1967. La réaction de Maillard. Description et répercussions physiologiques. Cah. Nut. Diet., $2(4), 3 \mathrm{r}-83$.

Pion R., I967. (Données non publiées.)

Pion R., Fauconneau G., I966. Les acides aminés des protéines alimentaires. Méthodes de dosage et résultats obtenus. Cah. A.E.C., 6, I55-175. 